\title{
Specific rumen microbiome traits predict average daily gain in beef cattle under different backgrounding systems
}

\section{Bobwealth 0. Omontese}

University of Minnesota Twin Cities Campus: University of Minnesota Twin Cities

Ashok K. Sharma

University of Minnesota Twin Cities Campus: University of Minnesota Twin Cities

Samuel Davison

University of Minnesota Twin Cities Campus: University of Minnesota Twin Cities

Emily Jacobson

University of Minnesota Twin Cities Campus: University of Minnesota Twin Cities

Megan J. Webb

University of Minnesota Twin Cities Campus: University of Minnesota Twin Cities

Andres Gomez ( $\nabla$ gomeza@umn.edu )

University of Minnesota https://orcid.org/0000-0002-1174-0368

\section{Research Article}

Keywords: Backgrounding systems, Beef cattle, Rumen microbiome, Average daily gain

Posted Date: September 27th, 2021

DOl: https://doi.org/10.21203/rs.3.rs-923855/v1

License: (9) (i) This work is licensed under a Creative Commons Attribution 4.0 International License.

Read Full License 
Specific rumen microbiome traits predict average daily gain in beef cattle under different backgrounding systems

Running title: Rumen microbiome during beef backgrounding

Bobwealth O. Omontese $^{1+}$, Ashok K. Sharma ${ }^{1}$, Samuel Davison ${ }^{1}$, Emily Jacobson ${ }^{1}$, Alfredo DiCionstanzo $^{1}$, Megan J. Webb ${ }^{1++}$ and Andres Gomez ${ }^{1 *}$

${ }^{1}$ Department of Animal Science, University of Minnesota, Saint Paul, MN 55108

+Current affiliation: Department of Food and Animal Sciences, Alabama A\&M University, Normal, AL 35762

++ Current affiliation: Community Engagement \& Partnerships, Eastern West Virginia Community and Technical College, Moorefield, WV 26836

*Corresponding author: gomeza@umn.edu 


\section{Abstract}

2 Background: Backgrounding $(\mathrm{BKG})$, the stage between weaning and finishing, significantly impacts feedlot performance in beef cattle; however, the contributions of the rumen microbiome

4 to this growth stage remain unexplored. A longitudinal study was designed to assess how BKG affects rumen bacterial communities and average daily gain (ADG) in beef cattle. At weaning, 38

6 calves were randomly assigned to three BKG systems for 55 days (d): a high roughage diet within a dry lot (DL, $n=13)$; annual cover crop within a strip plot $(\mathbf{C C}, \mathrm{n}=13)$; and perennial

8 pasture vegetation within rotational paddocks $(\mathbf{P P}, \mathrm{n}=12)$, as before weaning. After BKG, all calves were placed in a feedlot for $142 \mathrm{~d}$ and finished with a high energy ration. Calves were

10 weighed periodically from weaning to finishing to determine ADG. Rumen bacterial communities were profiled by collecting fluid samples via oral probe and sequencing the V4

12 region of the $16 \mathrm{~S}$ rRNA bacterial gene, at weaning, during BKG and finishing.

Results: Rumen bacterial communities diverged drastically among calves once they were placed

14 in each BKG system, including sharp decreases in alpha diversity for CC and DL calves only $(P$ $<0.001)$. During BKG, DL calves showed a substantial increase of Proteobacteria,

16 Succinivibrionaceae family (Ruminobacter, Succinimonas) $(\mathrm{P}<0.001)$, which also corresponded with greater ADG $(P<0.05)$. At the finishing stage, alpha diversity decreased dramatically and

18 Proteobacteria bloomed for all calves, with no previous alpha or beta diversity differences being retained between groups. However, at finishing, PP calves showed compensatory ADG,

20 particularly greater than that in calves coming from DL BKG, who showed the lowest ADG $(P=$ 0.02). Microbe network dynamics and network traits related to centrality, connectivity, degree,

22 number and strength of microbe-microbe interactions in the rumen were predictive of ADG during BKG and finishing.
\end{abstract}


24 Conclusions: Assessing rumen bacterial community composition, and particularly microbemicrobe interactions under different BKG systems may be useful in predicting growth

26 performance in beef cattle. These findings underscore the importance of early post weaning stages as potential targets for feeding interventions that can modulate the rumen microbiome to

28 enhance life-long productive performance in beef cattle.

30 Keywords: Backgrounding systems, Beef cattle, Rumen microbiome, Average daily gain

\section{Background}

The rapidly growing world human population requires more animal protein[1,2] and with the

34 U.S. population projected to increase $20 \%$ by 2050 [3], an additional production of 1.7 billion $\mathrm{kg}$ of beef will be required to meet future demands for animal protein. A major source of animal

36 protein in the U.S. comes from beef cattle[4] and as a result, producers continue to focus on improving cattle genetics and feed efficiency. Improving feed efficiency would boost the feed

38 utilization ratio, lower the amount of feed consumed and reduce environmental impacts of beef cattle production systems[5]. As such, understanding the dynamics of nutrient cycling from

40 feedstuff to the animal, across different animal developmental stages is critical to improve efficiency and sustainability of beef production systems[6-8].

42 Generally, commercial beef production systems comprise seedstock, cow-calf, backgrounding and feedlot components. Of these four segments, the backgrounding (BKG), the period between

44 calf weaning and placement into a feedlot[9], is vital. During this BKG period, which corresponds to the initial animal growth phase, a wide variety of feed resources are used to allow

46 for maximal body frame and minimal fat deposition; critical characteristics that predict animal efficiency and performance at finishing stages[10,11]. 
48 Several reports have focused on the influence of different feed sources during BKG and its duration on growth performance[12-15], highlighting diverse cost of gain and net return results

50 depending on the system used. For instance, many producers background calves by feeding a high roughage ration in a drylot (DL); however, this BKG system is associated with increased

52 labor and cost of deploying forage harvesting equipment[16,17]. Conversely, BKG calves by grazing standing summer grown perennial pasture (PP) may be a more economical option due to

54 reduced labor and greater compensatory gain when introduced to the feedlot[18]. On the other hand, BKG beef cattle on cover crops $(\mathbf{C C})$ comprises alternative forage sources for crop-

56 livestock production systems[11], which can protect the soil from erosion, improve nutrient cycling and increase soil productivity[19,20]. However, there are mixed results as far as the

58 efficiency and applicability of each BKG system.

One way to further understand efficiency and performance in beef production systems, in the

60 context of $\mathrm{BKG}$, is to characterize the composition and ecological interactions of the vastly diverse community of microbes that inhabit the animal rumen. This rumen microbiome,

62 significantly extends the physiological capabilities of the animal; by providing access to otherwise unavailable nutrients in feed, interacting with the animal metabolic and immune

64 landscape to impact health, and by defining the energetic efficiency and carbon footprint of the feeding process[21]. However, information on the effects of backgrounding on the rumen

66 microbiome and its relationship with animal performance, in response to diverse BKG systems, is scarce. Therefore, this study aimed to 1) determine if different BKG systems; CC, DL, PP, are

68 associated with specific rumen bacterial community composition from weaning to finishing in Angus and Angus x Simmental beef calves, and 2) investigate relationships between specific 70 microbiome traits and growth performance through BKG and finishing. 


\section{Results}

Following sequencing on the Illumina MiSeq platform, a total of 5,482,279 16S rRNA short

74 amplicon reads were obtained from 190 rumen fluid samples (amplicon libraries). After bioinformatic processing and read quality filtering, a total of 5,370,056 high quality reads

76 remained, with a mean sequencing depth of 29,006 reads/sample $(\mathrm{sd}=7,056.38)$ and a total of 4,149 taxonomically identified amplicon sequence variants (ASVs). From this procedure, a data

78 frame showing the abundance distribution of each ASV (as a proxy for bacterial species or strains) across 5 time points (T) from BKG to weaning was generated (Figure 1). Summary on

80 feed composition and nutrient analysis during the BKG and finishing phases of the study is summarized in Table 1.

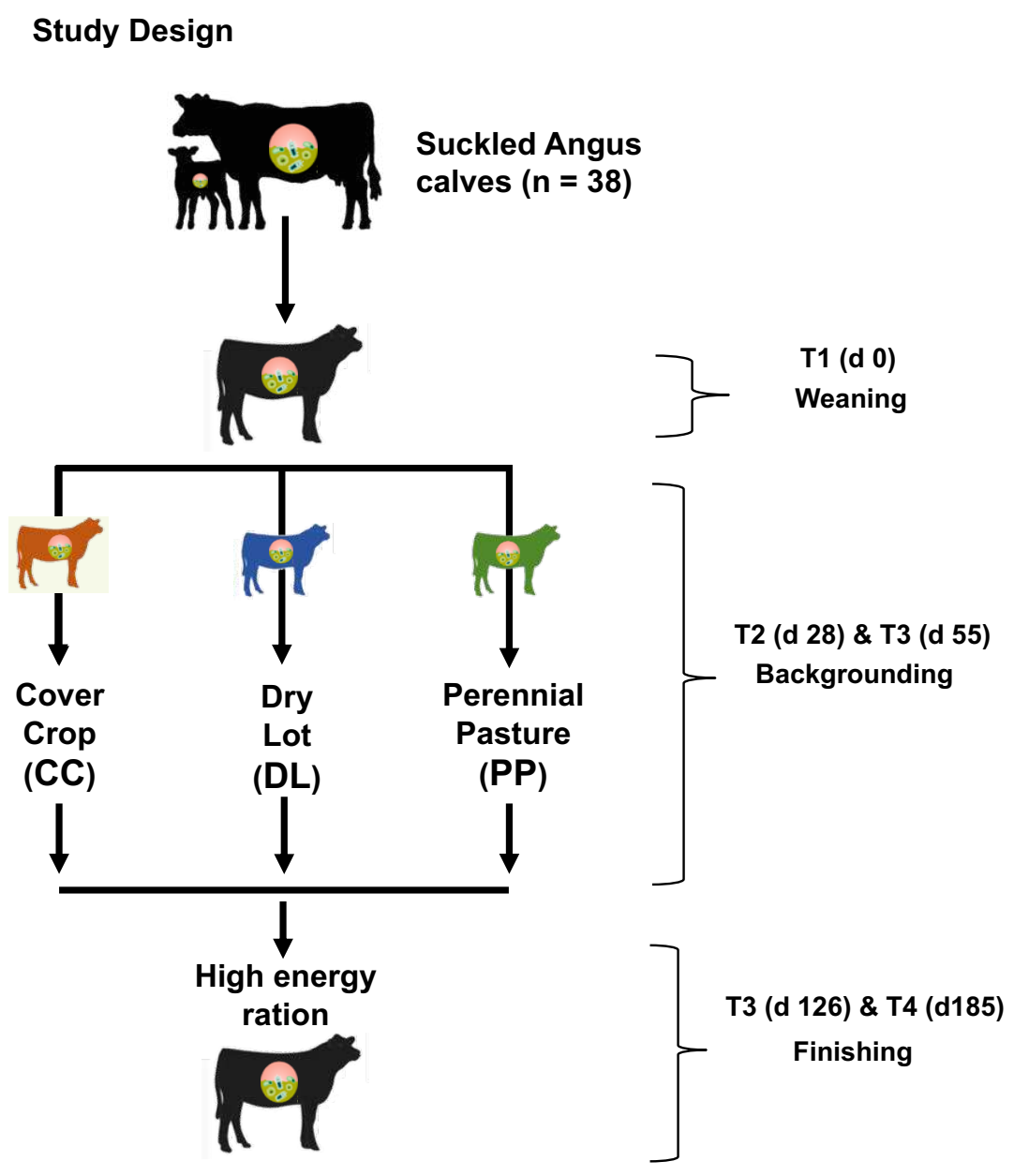


Fig. 1. Rumen fluid sampling protocol for 38 Angus and Angus x Simmental beef calves across five points

84 during backgrounding (BKG) and finishing. After weaning, the calves were randomly allocated to three different BKG systems: i) dry lot (DL, $n=13)$; ii) cover crop $(\mathrm{CC}, \mathrm{n}=13)$ and iii) a third group remained on grazing on

86 perennial pasture (PP, $n=12$ ), as before weaning. Rumen fluid samples were collected at weaning (T1), twice during BKG (T2 - T3), and twice at finishing (T4 - T5), when calves were kept on a high energy feedlot diet (FLD). Details 88 on the nutritional composition of BKG and FLD diets can be seen in table $\mathbf{1}$.

90 Table 1. Nutrient composition of backgrounding diet ( $\%$ of DM) fed to cattle

\begin{tabular}{|c|c|c|c|c|}
\hline \multirow{2}{*}{$\begin{array}{l}\text { Item } \\
\text { Nutrient composition }{ }^{3}\end{array}$} & \multicolumn{3}{|c|}{ Backgrounding ${ }^{1}$} & \multirow{2}{*}{$\begin{array}{r}\text { Finishing }^{2} \\
\text { ALL }^{2}\end{array}$} \\
\hline & DL & PP & $\mathrm{CC}$ & \\
\hline Moisture & 41.2 & 80.3 & 91.7 & 37.9 \\
\hline Dry matter & 58.8 & 19.7 & 8.3 & 62.1 \\
\hline NEm, Mcal $/ \mathrm{kg}$ & 1.6 & 1.5 & 1.6 & 1.9 \\
\hline Neg, Mcal/kg & 1.06 & 0.9 & 0.97 & 1.4 \\
\hline Starch & 31.4 & - & - & 39.6 \\
\hline NDF & 33.4 & 47.7 & 28.1 & 25.3 \\
\hline $\mathrm{CP}$ & 12.6 & 21.3 & 19.5 & 13.4 \\
\hline Fat & 4.3 & 3.6 & 2.6 & 4.9 \\
\hline $\mathrm{Ca}$ & 0.6 & 0.6 & 1.3 & 0.6 \\
\hline $\mathrm{K}$ & 1.8 & 2.6 & 4.1 & 1.0 \\
\hline
\end{tabular}




\begin{tabular}{|c|r|r|r|r|}
\hline $\mathrm{Mg}$ & 0.2 & 0.2 & 0.2 & 0.2 \\
\hline $\mathrm{S}$ & 0.1 & 0.2 & 0.5 & 0.2 \\
\hline
\end{tabular}

$92{ }^{1}$ During backgrounding, animals were allocated to either one of three treatments; DL (calves were fed a haylage ration in dry lot), PP (calves grazing perennial pastures) or CC (calves grazing summer grown cover crop) for $55 \mathrm{~d}$.

$94{ }^{2}$ During finishing, all animals received four concentrate-adaptation diets over a period of 28 days.

${ }^{3}$ Calves received free-choice minerals (Wind \& Rain, Purina Animal Nutrition LLC, MN) during the backgrounding

96 phase and nutrient analysis conducted on weekly feed samples.

${ }^{4} \mathrm{ALL}=$ calves backgrounded in CC, DL and PP were fed a similar high energy ration.

\section{Broad taxonomic composition of rumen bacteria in beef calves during backgrounding}

\section{0 (BKG) and finishing}

Rumen bacterial communities in the 38 calves during backgrounding (BKG) and finishing were

102 mainly assigned to seven broad taxonomic groups: Bacteroidetes (37\%) and Firmicutes (35\%)

were the two most abundant phyla, followed by Proteobacteria (12\%), Verrucomicrobia (3\%),

104 Actinobacteria (2\%), Tenericutes (1.5\%), and SR1 (1.2\%). Euryarchaeota (4\%) and

Crenarchaeota $(<1 \%)$ were the two archaeal phyla detected. However, significant distinctions in

106 the abundance distribution of specific phyla between calves on each BKG system and at finishing were evident (Figure 2a). For instance, one of the main distinctive taxonomic traits

108 observed during BKG, was the predominance of the phylum Proteobacteria in DL calves $(9.24 \%$ $\pm 5.15)$, compared with calves in $\mathrm{CC}(2.19 \% \pm 0.61)$ and $\mathrm{PP}(2.23 \% \pm 0.52)$ (Figure $2 \mathbf{b}$ and c)

110 Kruskal-Wallis, $P=1.6 \mathrm{e}-10)$. It was also noted that Proteobacteria, was predominant at the finishing stage (T4 and T5) compared to BKG in all three groups; however, the abundance of 
112 Proteobacteria remained the highest in DL calves during this stage (Kruskal-Wallis, $\mathrm{P}=0.02$,

Figure 2c).

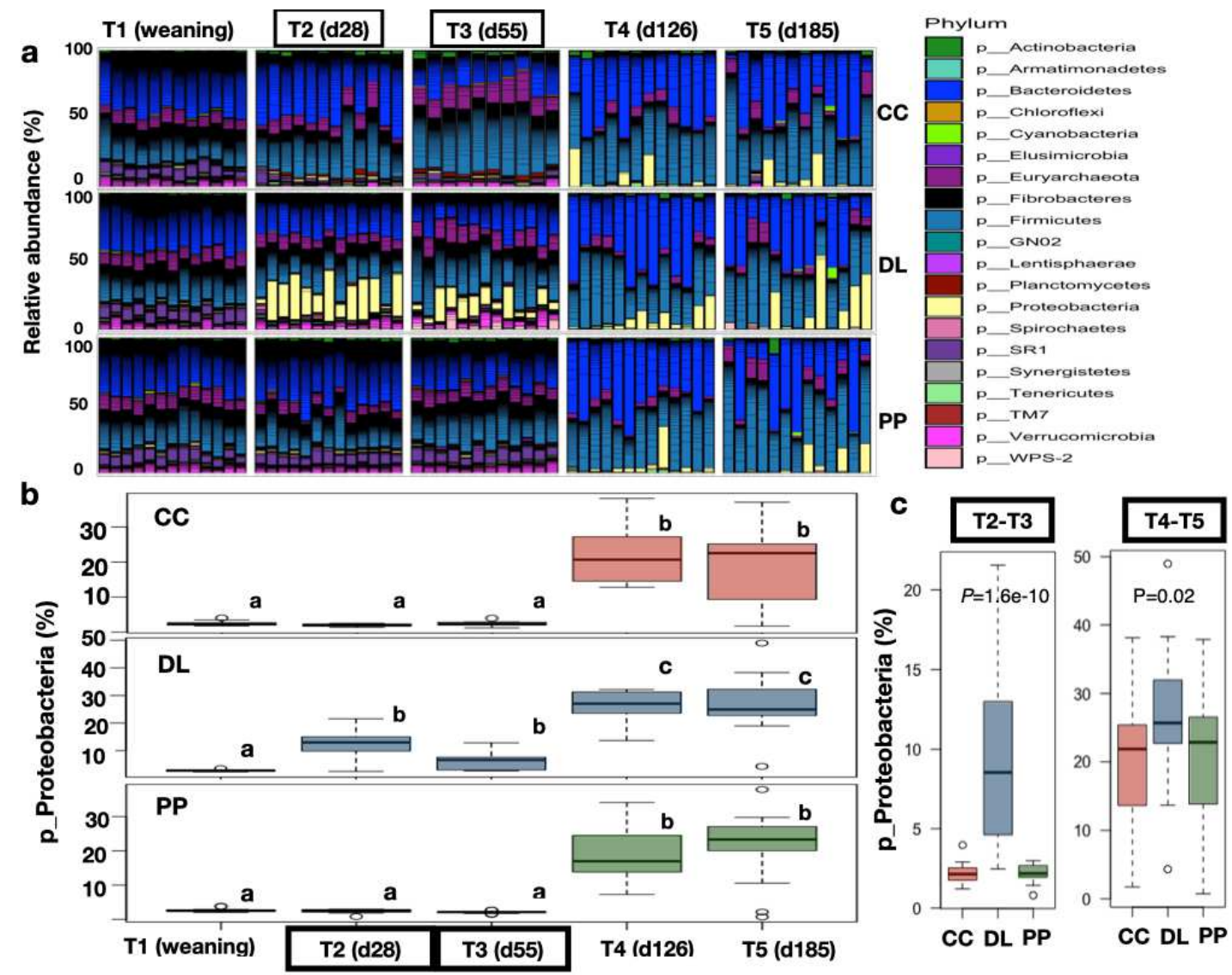

Figure 2. Taxonomic composition at phylum level in the rumen microbiome of calves at weaning (T1),

116 backgrounding (T2 -T3), and finishing (T4 -T5). (a) Barplot showing the relative abundance of phyla at weaning, backgrounding and finishing. (b) Boxplots showing relative abundances of Proteobacteria in calves within every

118 BCK system, from weaning to finishing. (c) Boxplots showing relative abundances of Proteobacteria in calves within time point, BCK:T2-T3 and finishing: T4-T5. Dry lot (DL); cover crop (CC) and perennial pasture (PP).

\section{Rumen bacterial diversity was significantly impacted by backgrounding and finishing}

122 Alpha diversity analyses revealed significant changes in bacterial diversity during backgrounding and finishing. After weaning, for calves moved to $\mathrm{CC}$ and DL BKG (T2 and T3), the number and proportion of different bacterial strains or species (observed ASVs and Shannon diversity index) 
decreased significantly (Kruskal-Wallis test, $P<0.001$ ), while remaining relatively stable for PP

126 claves. This pattern was expected as PP calves remained on the same diet after weaning and during BKG. However, once DL, CC and PP calves were moved to the finishing phase (T4 and 128 T5), alpha diversity decreased sharply (Kruskal-Wallis test, $P<0.001$ ), with no differences detected between BKG groups, at early or late finishing (Figure 3a and b).

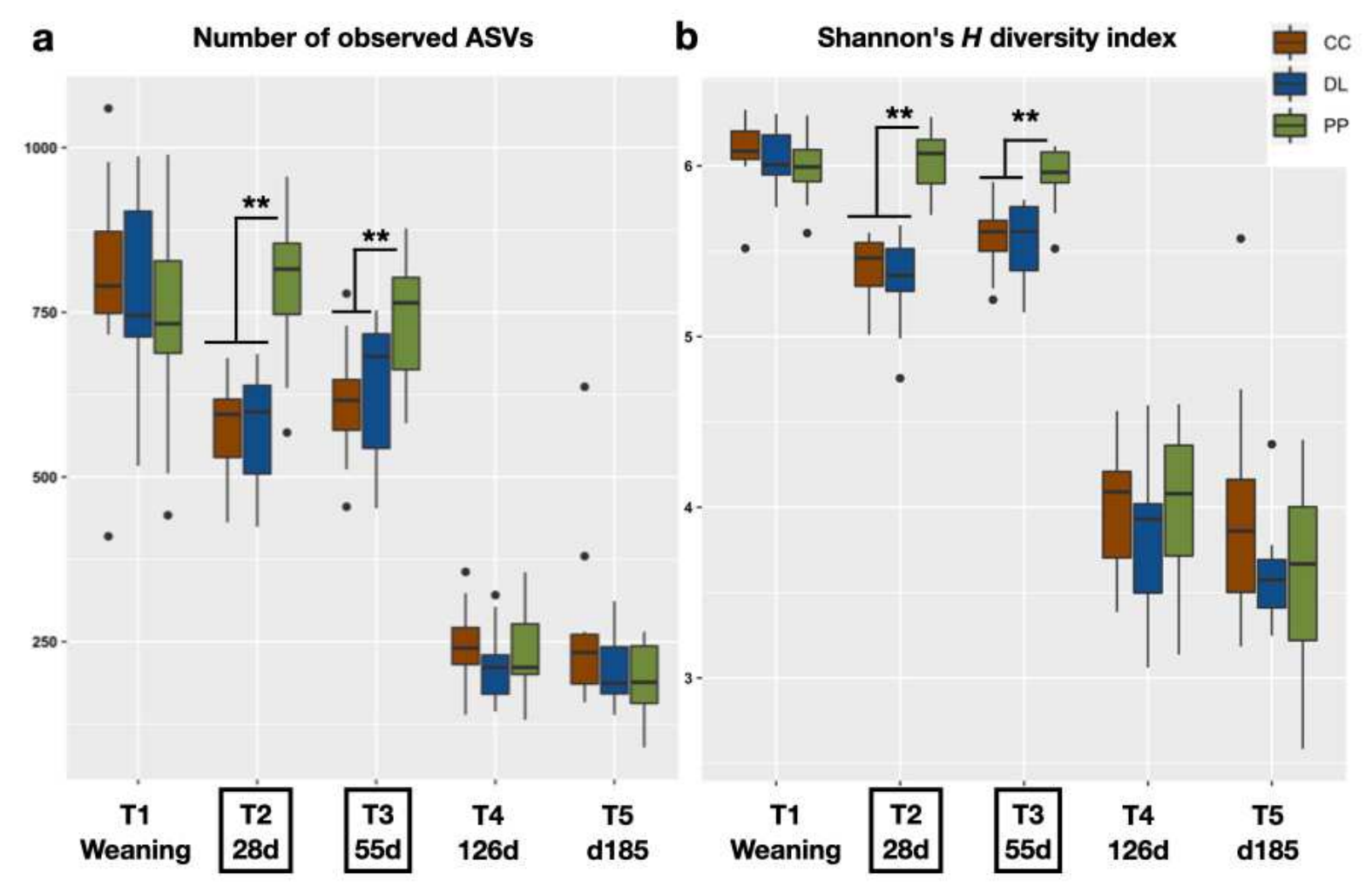

Fig 3. Rumen bacterial alpha diversity at weaning (T1), backgrounding (T2 -T3), and finishing (T4 -T5). (a)

132 The number of different ASVs detected decreased significantly for calves moved to DL and CC BKG, but remained stable for calves on PP during this period. This number decreased again for all BKG groups at the finishing stage. (b)

134 The patterns observed with the number of different ASVs as shown in (a) were replicated when measuring the Shannon index of diversity, which not only takes into account presence or absence of different ASVs, but also their abundance 136 distribution. Dry lot (DL); cover crop (CC) and perennial pasture (PP). Asterisks show significant differences based on Kruskal-Wallis tests adjusted for multiple comparisons $\left(* * \mathrm{P}<0.001,{ }^{* * *} \mathrm{P}<0.0001\right)$. 


\section{Different BKG systems and the finishing stage are associated with unique rumen}

\section{0 microbiome profiles}

Next, similarities and differences in the presence of specific rumen bacterial taxonomic groups

142 (ASVs) and their relative abundances (\%), during BKG and finishing were considered. This beta diversity analysis showed that, after weaning [day 28 (T2) and day 55 (T3)], each BKG system

144 was characterized by very unique rumen microbiome profiles (Figure 4a, ANOSIM's R>0.9; PERMANOVA's R2 $>0.4$ and F>11.7, $P<0.001$ ). However, once at finishing [day 126 (T4),

146 day 185 (T5)], these differences were not maintained, likely reflecting that all calves were under the same high energy feedlot diet.

148 The data also show that although the major dietary changes characterizing BKG and finishing had the strongest effect on the rumen microbiome of all calves (PERMANOVA's R2>0.43 and

$150 \mathrm{~F}>23, P<0.001$ ), intrinsic drivers such as developmental stage or age were also significant factors, particularly during finishing (Figure S1). For instance, although it seemed that the

152 rumen microbiome of calves remained unchanged under the same diet during BKG, closer inspection of the data shows significant compositional shifts from day 28 to 55 during this stage

154 (ANOSIM's $\mathrm{R}>0.65, \mathrm{P}<0.001$ ), with less pronounced changes from day 126 to 185 at finishing, particularly for PP claves (ANOSIM's $\mathrm{R}=0.16-0.21, \mathrm{P}=0.006-0.02$ ) (Figure S1a). In addition, it

156 was observed that the rumen microbiome of calves exhibited increased interindividual variability as they got older, with higher heterogeneity in microbiome profiles between individuals at the

158 finishing stage (day 126-185) (Kruskal-Wallist test $\mathrm{P}<0.05)$ (Figure S1b).

Subsequently, we sought to mine for taxonomic features characterizing each BKG system. A

160 species indicator analysis was used to mine for specific ASVs that were unique to, and more abundant in each BKG group once calves were weaned (T2 -day 56, Indicator value $>0.7$, FDR-

162 adjusted, Kruskal-Wallis test; $\mathrm{P}<0.001$, table S1). These analyses revealed that 54, 42 and 78 
indicator ASVs faithfully characterized calves on CC, DL and PP BKG respectively. Although

164 the majority of indicator ASVs across all three BKG systems were affiliated to the Clostridiales and Bacteroidetes orders (mainly Prevotellaceae and Lachnospiraceae families), the proportions

166 of these taxa were different in each system, with other taxonomic groups also showing unique patterns (Figure 4b, Table S1).

168 For instance, most indicator ASVs characterizing calves under CC BKG were largely affiliated to the Clostridiales (unclassified, Lachnospiraceae), with contributions from ASVs classified as

170 Methanosphaera (Archaea-Methanobacteriales), Coriobacteraceae (Actinobacteria), Sphingomonas, Desulfobulbus, Succinivibrionaceae (Proteobacteria), and Mollicutes (order

172 RF39). The vast majority of indicator ASVs characterizing calves under PP BKG were largely assigned to the Bacteroidales order (unclassified, BS11 and Prevotellaceae), also with

174 contributions by ASVs classified as Methanomassiliicoccaceae (the vadinCA11 genus), Deltaproteobacteria (orders GMD14H09, Myxococcales, ands PB19), Mollicutes (unclassified

176 Anaeroplasmataceae, Anaeroplasma and 4 ASVs from the RF39 order) and Verrucomicrobia (RFP12 order).

178 Calves under DL BKG were characterized by lower abundance of Clostridiales, but particularly, by higher abundances ASVs from the Succinivibrionaceae family (Anaeromonadales order,

180 Phylum Proteobacteria); specifically, Ruminobacter, and Succinimonas were significantly enriched in DL calves compared to any other group during BKG (Figure S2). Other indicator

182 taxa distinguishing DL BKG were from the Spirochaetales order (specifically, unclassified Treponema), unclassified Erysipelotrichaceae (RFN20), Verrucomicrobia (RFP12) and ASVs

184 from the order Synergistales and TM7 (F16 family). Figure 4b shows that the cumulative relative abundance of these indicator taxa (classified at the order level) peaks at day 28 (T2), the 186 first BKG time point analyzed, diminishing sharply through day 55 of BKG and finishing, except 
for indicator ASVs for calves under DL BKG, probably due to the prevalence of Aeromondales
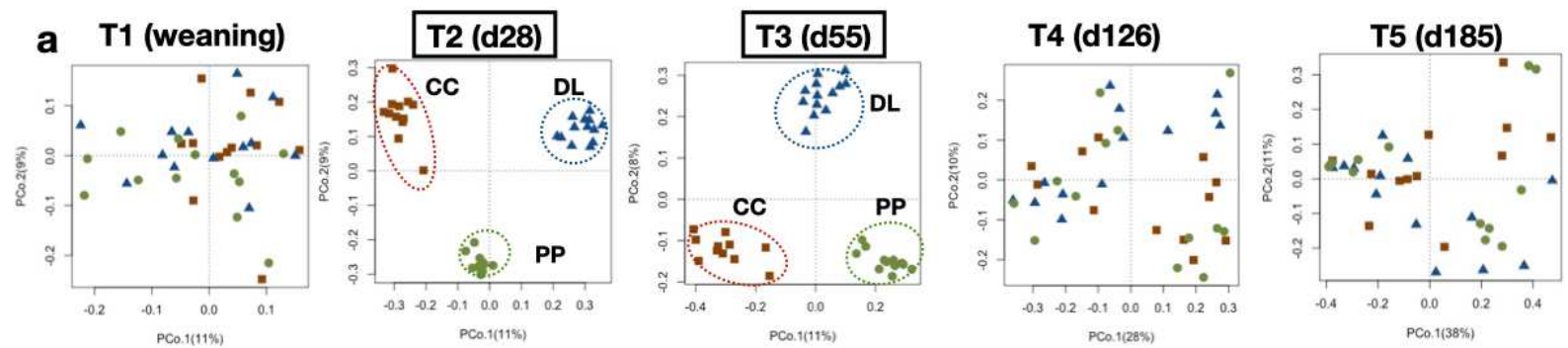

\begin{tabular}{|cc|cc|}
\hline \multicolumn{2}{|c|}{ ANOSIM } & \multicolumn{2}{|c|}{ PERMANOVA } \\
\hline R & 0.05 & R2 & 0.06 \\
P & 0.05 & F & 1.19 \\
& & P & 0.05 \\
\hline
\end{tabular}

\begin{tabular}{|cc|cc|}
\hline \multicolumn{2}{|c|}{ ANOSIM } & \multicolumn{2}{|c|}{ PERMANOVA } \\
\hline $\mathbf{R}$ & 0.97 & $\mathbf{R 2}$ & 0.42 \\
$\mathbf{P}<$ & 0.001 & $\mathbf{F}$ & 13.05 \\
& & $\mathbf{P}<$ & 0.001 \\
\hline
\end{tabular}

\begin{tabular}{|cc|cc|}
\hline \multicolumn{2}{|c|}{ ANOSIM } & \multicolumn{2}{|c|}{ PERMANOVA } \\
\hline R & 0.95 & R2 & 0.4 \\
P< & 0.001 & $\mathbf{F}$ & 11.7 \\
& & P< & 0.001 \\
\hline
\end{tabular}

\begin{tabular}{|cc|cc|}
\hline \multicolumn{2}{|c|}{ ANOSIM } & \multicolumn{2}{|c|}{ PERMANOVA } \\
\hline R & 0 & R2 & 0.04 \\
P & 0.4 & F & 0.89 \\
& & P & 0.5 \\
\hline
\end{tabular}

\begin{tabular}{|cc|c|c|}
\hline \multicolumn{2}{|c|}{ ANOSIM } & \multicolumn{2}{|c|}{ PERMANOVA } \\
\hline R & 0 & R2 & 0.04 \\
P & 0.5 & F & 0.86 \\
& & P & 0.54 \\
\hline
\end{tabular}

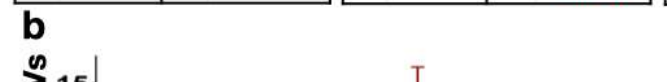

\section{Indicator taxa}
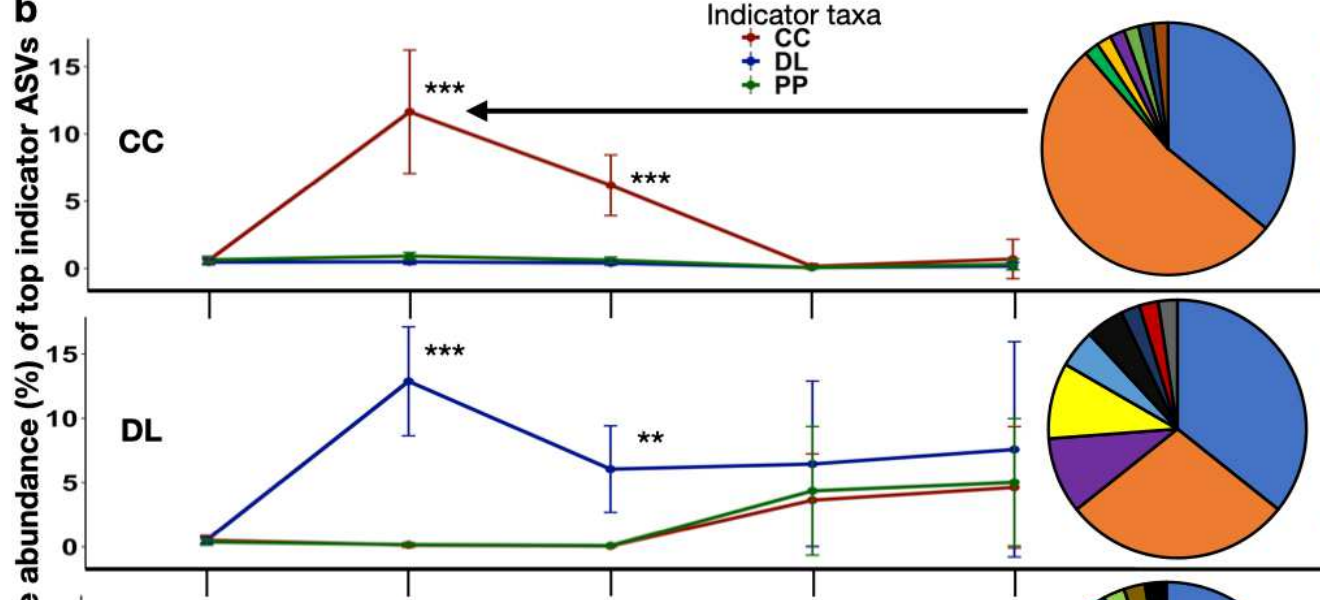

Order

aBacteroidales

口Clostridiales

口Sphingomonadales

口Desulfobacterales

口Aeromonadales

口Tenericutes

-Methanobacteriales

口Coriobacteriales
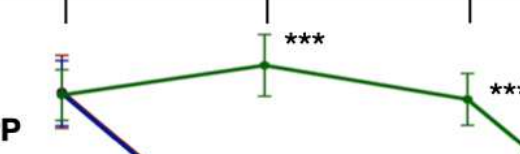

PP
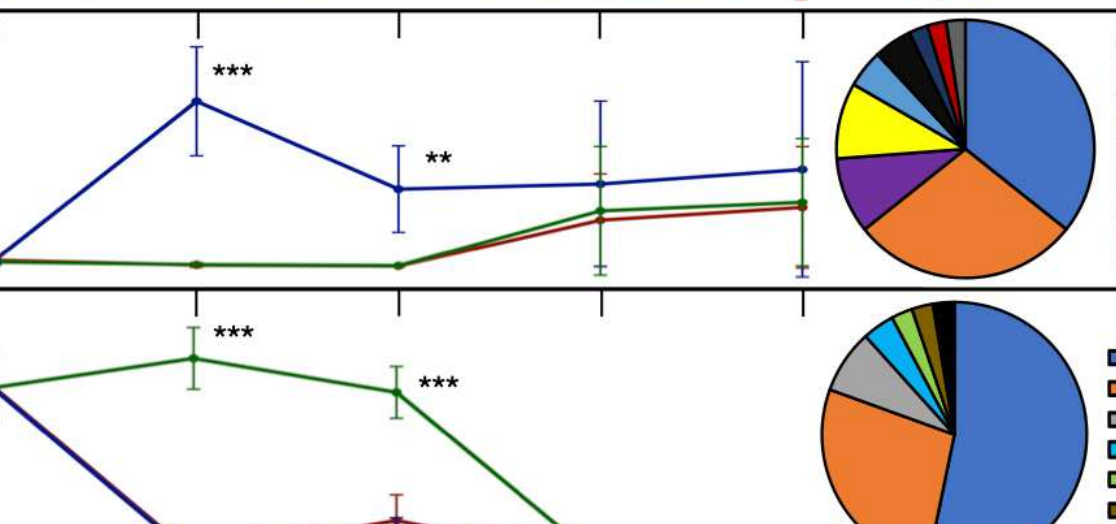

口Bacteroidales

口Clostridiales

-Aeromonadales

$\square$ Spirochaetales

口Erysipelotrichales

- Verrucomicrobia

-Fibrobacterales

a Synergistales 口TM7

Figure 4. Rumen bacterial composition and abundance of indicator taxa at weaning (T1), backgrounding (T2

-T3), and finishing (T4 -T5). (a) Principal coordinate analysis (PCoA, Bray-Curtis distances) showing bacterial compositional differences at weaning, BKG and finishing. ANOSIM and PERMANOVA test results, along with 
BKG system, at weaning, BKG and finishing. The line plots show how the cumulative abundance of these indicator

198 ASVs peaks during BKG (T2), the piues show the taxonomic affiliation, at the order level., of the indicator ASVs.Asterisks show significant differences in the abundance of indicator taxa between groups based on Kruskal-

200 Wallis tests adjusted for multiple comparisons (**P<0.001, ***P<0.0001). Dry lot (DL); cover crop (CC) and perennial pasture (PP)

\section{Calves in under BKG system display unique co-abundance network patterns}

204 The manner in which individual bacterial species co-abound or interact within a microbiome could be used as a proxy for the functional relevance and potential metabolic contribution of

206 microbes in a given system[22,23]. With that premise, we characterized co-abundance dynamics of specific rumen bacterial taxa (ASVs) when calves were moved from weaning to each BKG

208 system and to the finishing stage, and inferred their collective functional potential using networktheory analyses[24]. For instance, at weaning, the rumen microbiome of all 38 calves showed

210 extremely low connectivity (or number of interactions), with only 11 sparse interactions detected (compositionally corrected Spearman's correlation coefficient $>0.7, \mathrm{q}<0.05)($ Figure 5a). This

212 observation is likely a reflection of a very immature rumen microbiome, when reliance on lactation still constitutes an important feeding source. However, when calves were moved to

214 each BKG system, interactions between rumen bacteria became more complex (from 193 to a maximum of 545 interactions at day 56 of BKG for DL calves, Figure 5a and S3).

216 To investigate if these complex rumen bacterial interactions are unique to each BKG system, we measured and compared several network attributes across BKG and finishing. For example,

218 figure 5a and $\mathbf{b}$ show that average neighbor connectivity and degree, two centrality measures denoting the number of local and wide (direct/indirect) interactions between rumen bacteria[25], 220 were significantly higher for calves on DL and lowest in calves on PP, particularly at day 56 of 
BKG (T3) (Kruskal-Wallis multiple comparisons, $\mathrm{P}<0.001$ ). Conversely, the average shortest

222 path length, a proxy for functional distance between bacterial taxa (or how fast information moves through a network [26]), was always the lowest in DL calves and the highest under PP

224 BKG at day 28 (T2) and 56 (T3).

At finishing (days 126 and 185), although all calves were under the same high energy diet, and

226 alpha and beta diversity patterns distinguishing groups during BKG were not conserved, network dynamics were also significantly different, not only compared to the BKG stage, but also among

228 calves coming from each BKG system (Figure 5). For instance, although the number of rumen bacterial interactions decreased dramatically under the finishing diet in all calves coming from

230 CC, DL and PP BKG ( 35 in DL calves to around 50 for CC and PP groups, Figure S3), the patterns observed at BKG were reversed; specifically, the rumen microbiome of DL calves

232 showed the lowest average neighbor connectivity and degree centrality, and the highest average shortest path length compared to PP and CC calves (Figure 5a and b). Overall network topology

234 traits, considering these and other network attributes simultaneously (closeness centrality, clustering coefficient, eccentricity and radialty), showed similarities between CC and PP calves

236 during BKG and finishing, which distinguish them from all network attributes observed in DL calves, particularly at day 56 (T3) (Figure S4a).

238 Next, we mined for the most significant interaction patterns between taxa, measured as the average strength of correlation (Spearman Rho coefficients) and average number of shortest

240 paths that go through a given interaction (Edge betweenness). These analyses revealed that although average correlation strength between bacterial taxa (Spearman's rho) was largely 242 similar in CC, DL and PP claves during BKG (mean: 0.75-0.77,), rumen bacterial communities in PP claves showed the greatest correlation strength at finishing (Figure S4b). In line with the 244 average shortest path length patterns mentioned above, edge betweenness was the lowest in DL 
calves during BKG (d28 and d56); but at finishing, it was PP calves that showed the lowest

246 number of shortest paths that go through a given interaction.

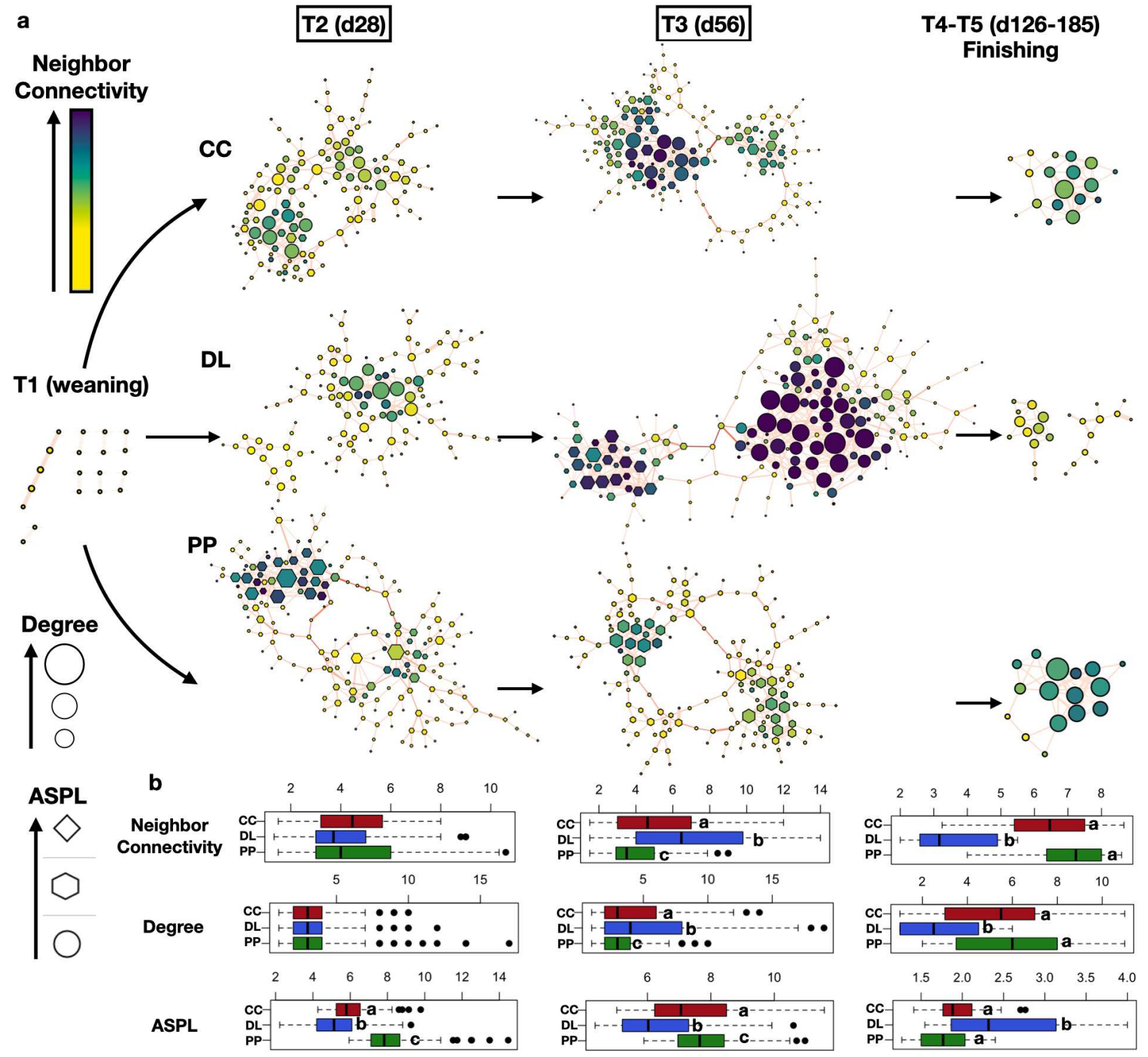

Figure 5. Co-abundance networks of rumen bacterial taxa at weaning (T1), backgrounding (T2 -T3), and

250 finishing (T4 -T5). (a) Network topology and attributes in the rumen microbiome of calves at weaning, BKG and

finishing can be visualized. Nodes represent a given ASV and edges show the association (correlation) in abundance

252 between two given bacterial taxa (nodes). Color represents neighbor connectivity, which measures the average

connectivity of all surrounding nodes in the network. Size of node represents the average degree of connectivity or 
254 number associations a given node has in the network. Shape represents the average shortest path length, a measure of how fast information can travel through a network. Differences in the variation of all these network attributes

256 between each group at BKG and finishing can be observed in the box plots depicted in panel (b), where letters represent significant differences based on Wilcoxon Rank Sum tests $(\mathrm{P}<0.05)$. Dry lot (DL); cover crop (CC) and 258 perennial pasture (PP)

\section{Average daily gain (ADG ) differed in each BKG system and at finishing}

During BKG, ADG was the greatest $(P<0.05)$ in DL calves, particularly compared with calves

262 under PP (1.4 vs $0.9 \mathrm{~kg} / \mathrm{d})$. Indeed, PP claves showed the lowest ADG during this stage.

However, during the finishing stage, PP calves showed a compensatory ADG, with significantly

264 higher values compared with DL calves ( $1.9 \mathrm{vs} 1.6 \mathrm{~kg} / \mathrm{d}, P=0.02$, ). Overall, during finishing, ADG was greater in PP calves $(1.6 \mathrm{~kg} / \mathrm{d})$, followed by CC claves $(1.5 \mathrm{~kg} / \mathrm{d})$, with calves under 266 DL showing the lowest values $(1.4 \mathrm{~kg} / \mathrm{d})$, when averaging all data across all study stages (Table 2).

\begin{tabular}{|c|c|c|c|c|}
\hline \multirow[b]{2}{*}{ ADG } & \multicolumn{4}{|c|}{ Treatment ${ }^{1}$} \\
\hline & DL & PP & $\mathrm{CC}$ & $P$-value ${ }^{2}$ \\
\hline Backgrounding & $1.4 \pm 0.1^{\mathrm{a}}$ & $0.9 \pm 0.1^{\mathrm{b}}$ & $1.2 \pm 0.1^{\mathrm{ab}}$ & $<0.001$ \\
\hline Finishing & $1.6 \pm 0.1^{b}$ & $1.9 \pm 0.1^{\mathrm{a}}$ & $1.7 \pm 0.1^{\mathrm{ab}}$ & 0.017 \\
\hline Overall & $1.4 \pm 0.0^{\mathrm{a}}$ & $1.6 \pm 0.0^{b}$ & $1.5 \pm 0.0^{\mathrm{ab}}$ & 0.021 \\
\hline
\end{tabular}


${ }^{1}$ Calves were stratified by birth date, birth weight, gender and dam age to 1 of 3 treatments: 1 ) dry lot (DL, calves

272 were fed a haylage ration in a dry lot); perennial pasture ( $\mathrm{PP},=$ calves grazing perennial pasture); and cover crop (CC, calves grazing summer grown cover crop) for $55 \mathrm{~d}$.

$274 \quad{ }^{2}$ Probability of a difference among least squares means.

${ }^{3}$ Measurements indicated are averages of data collected over the entire study period.

278 Discussion

Although others have demonstrated the impact of backgrounding systems on performance

280 characteristics of beef cattle[12-14], this was the first study, to the best of our knowledge, to evaluate the effects of backgrounding on rumen microbiome structure from weaning to finishing.

282 This report demonstrates that rumen bacterial profiles in BKG predict ADG at both BKG and finishing. We place importance on the influence of rumen bacterial communities on growth

284 performance, not only in terms of the possible contributions of specific rumen bacterial taxa, but particularly, on how interactions between rumen bacteria impact performance depending on the

286 BKG system selected.

288 The rumen microbiome is unique under specific BKG systems and finishing in beef cattle

During growing stages, the rumen microbiome plays a critical role in feed efficiency by

290 harnessing energy from feed for muscle development, through fermentation and protein synthesis[8,27]. In many beef production systems, BKG is characterized by a change from calves

292 suckling dams in addition to grazing pastures to a high-fiber, low-energy diet before final transition to a feedlot where calves receive a low-fiber, high-energy diet [28]. However, 294 depending on the specific BKG diet, different rumen microbial profiles and fermentation 
activities would be expected, which may have a direct impact on growth performance and 296 efficiency[29,30].

In this study, it was clear that each BKG system resulted in unique rumen bacteria profiles after

298 weaning. In fact, specific taxonomic groups dominated when calves faced particular dietary challenges in BKG and finishing, causing a sharp drop in overall alpha diversity (Figures $\mathbf{2}$ and

300 4). This drop in diversity is attributed to the specialized diets provided during BKG and finishing, each characterized by the availability of specific feed substrates[31,32], triggering the

302 bloom and/or the suppression of particular taxa that compete for preferred and/or available energy sources.

304 One of the most remarkable observations was the bloom in Succinivibrionaceae observed in calves under DL BKG, specifically the increase in Ruminobacter and Succinimonas genera

306 (Figure S2, 2 and 4). Succinivibrionacae are reported to be core taxa in beef cattle and one of the most active bacteria during growing and finishing stages[8,33]. Members of this family, 308 including Selenomonas, and Succinivibrio, have been observed to increase in the rumen of steers when suppressing methanogens using encapsulated nitrates, and in positive association with high 310 energy diets, ADG and feed efficiency[34-37]. Succcinivibrinaceae are a known amylolytic bacterial group, which have an increased capability

312 to produce succinic acid and formate from glucose fermentation; they can also utilize $\mathrm{CO} 2$ as main substrate, favoring the production of propionate, which as been associated with higher feed 314 efficiency ruminants [37-39]. Such metabolic outcomes can also positively influence efficiency of the fermentation process by altering electron sinks and antagonizing with methanogenes in the 316 rumen[40]. These observations are consistent with the higher starch content of the DL diet, which appears to be rapidly fermented in the rumen[38]. Thus, the availability of a readily 
318 degradable starch in DL could have favored greater ADG observed in these calves during BKG, mediated by the blooms in Succinivibrionaceae and their metabolic products.

\section{Bacterial co-abundance network traits predict ADG at BKG and finishing in beef cattle}

322 An increase in highly and readily degradable starch in the rumen of DL calves could also explain the unique co-abundance network patterns observed in this group during $\mathrm{BKG}$, in contrast with

324 CC and PP calves (Figure 5). Analyses of network centrality measures can denote the ability of microbial communities to functionally respond to external and internal stimuli[22,23], in this

326 case substrate availability. As such, a high starch degradation rate in DL calves may have altered metabolic interactions between rumen taxa, which upon release of specific metabolic products

328 (e.g. Succinivibrionaceae-derived formate or succinic acid) could have increased the number of metabolic associations surrounding a given taxon (neighbor connectivity and total number of

330 interactions), boosted the number of metabolic interactions of a given taxon (degree) and decreased the number of interactions or steps that would take to metabolically connect all

332 possible pairs of rumen taxa (edge betweenness and path length) (Figure 5 and S5). According to concepts in network theory, the microbiome of DL calves could have been more efficient in

334 transporting information throughout microbial metabolic networks in the rumen during BKG, likely positively influencing ADG at this stage.

336 Thus, it can be hypothesized that microbial interactions, and hence microbiome network traits in the rumen, can predict animal physiological performance more accurately than the presence,

338 bloom or suppression of specific taxa. For example, calves under PP BKG not only had the lowest BKG ADG, but also opposite network traits compared to those seen in DL calves during 340 BKG. However, once at the finishing stage, when all alpha and beta diversity traits were largely the same for all groups and despite the fact that all calves were under the same high energy diet, 
342 PP calves not only showed greater ADG (significantly higher than DL calves), but also greater interaction strength, lowest path lengths and edge betweenss (Figure 5 and S5). These traits

344 were also observed despite the fact that DL calves retained the highest abundance of Succcinivibroinaceae at finishing, which likely influenced the higher ADG in these calves during 346 BKG (Figure S2).

The greater ADG of PP calves during finishing can be attributed to compensatory gain resulting

348 from initial slower growth of PP calves during backgrounding. Research suggests that compensatory growth is influenced by nutrient restriction, including type of nutrient being

350 restricted, the length of nutrient restriction and the type of diet fed following restriction[41]. Our data reveal that microbiome composition, and particularly, the specific characteristics of

352 microbe-microbe interactions or network traits are also associated with compensatory weight gain. Thus, during BKG, the rumen of PP calves could have exhibited smaller net energy

354 available to rumen microbes due to the high fiber content in pastures and restriction of starch (Table 1), leading to less dynamic rumen microbiome networks. This observation supports the 356 dominance of Bacteroidales (Prevotellaceae) in PP calves during BKG, a taxonomic group reported to display greater metabolic versatility in ruminants when tackling high fiber diets, but 358 that exhibits different metabolic outputs compared with taxa better suited to metabolize high energy diets (e.g. Succinivibrionaceae)[21,27,42,43].

360 Therefore, an adaptation to high fiber diets in PP calves during BKG, including a slower metabolic turnover in the rumen, could have programmed their rumen environment to maximize 362 energy harvest and growth at finishing. The mechanisms behind these delayed responses are unclear, but high forage during BKG, at the expense of high energy for growth, could have

364 enhanced health by positively modulating the epithelial structure of the host rumen mucosa[44]. A high fiber diet during BKG could have also triggered a carryover effect on the rumen 
366 microbiome, preparing PP calves to better tackle high energy diets potentially prone to metabolic distress at finishing $[31,33,45]$. Based on the observation that the network attributes of $\mathrm{CC}$ and

368 CC calves and ADG patterns were more similar during BKG (Table 2 ands Figure S5), compared to those seen in DL calves, it can be speculated that a forage based diet during this

370 growing stage, either based on perennial pastures or cover crops, achieves analogous microbiome modulation and physiological outcomes in beef cattle at finishing, independent from taxonomic

372 assortment.

\section{Study limitations}

The main limitation of our study is the absence of data supporting actual metabolic responses to

376 each of the three BKG systems. As such, the results obtained should be validated using actual functional data from the rumen microbial communities evaluated, included but not limited to

378 metabolomic and metagenomics approaches at BKG and finishing. However, these compositionally-based results reflect previous data on the associations between active rumen 380 taxa, as measured by RNAseq, and physiological performance in beef cattle[46,47]. Shorter rumen fluid collection interval following allocation into different backgrounding systems and

382 during the finishing phase could also provide a better understanding of how rumen microbial communities, and associated metabolic products, begin to change under the influence of specific

384 BKG diets. Finally, we also acknowledge that although the per-group sample sizes considered herein are customary of microbiome studies of similar scope[8,31,48], the performance traits reported such as $\mathrm{ADG}$, in the context of microbiome composition and rumen metabolites during BKG, should be evaluated and validated based on large within group populations.

\section{Conclusions}


390 Our data show that specific rumen microbiome traits, and particularly, patterns of interactions between rumen taxa, can predict growth performance in beef cattle at BKG and finishing stages.

392 Specifically, increasing dietary energy during BKG may only temporarily affect energetic turnover in rumen microbial populations and growth performance. In contrast, keeping calves

394 under more cost-effective BKG systems such as pastures or cover crops, which enhances fiber degradation during $\mathrm{BKG}$, may prove to be more effective on growth performance in the long run.

396 The implication of these results is that producers could employ targeted feeding strategies at early life to modify the rumen microbiome of their herd and program feed efficiency in

398 subsequent production stages. However, it is likely that the window for microbiome modulation needs to be carefully selected, given the dynamic nature of the rumen microbiome in early

400 developmental stages, even when calves are under the same diet (Figure S1) Finally, these data highlight the need to focus attention beyond taxonomic markers of animal performance, to focus 402 on metabolic interactions between taxa and microbial network traits as more accurate markers of physiological performance in microbiome studies focusing on animal production systems.

\section{Materials and methods}

\section{Animals and Experimental Design}

All animal care and experimental protocols were approved by the University of Minnesota

408 Animal Care and Use Committee (approval number 1807-36177A). This study was conducted at the North Central Outreach Research Station (NCROC), University of Minnesota, Grand Rapids,

410 MN. A total of 38 Angus and Angus $x$ Simmental beef calves comprising steers $(n=18)$ and heifers $(n=20)$ were enrolled in the study. Cow-calf pairs grazed a mix of introduced pasture

412 grasses typical of a Northern mixed prairie. A completely randomized design was used to stratify 
calves by dam age, birth date, birth weight, and sex to 1 of 3 backgrounding systems for $55 \mathrm{~d}$

414 after weaning: 1) fed a high roughage ration delivered in a dry lot (DL); 2) grazing perennial pasture vegetation (PP), and 3) grazing summer grown cover crop (CC). All experimental

416 animals received a free-choice mineral (Wind and Rain, Purina Animal Nutrition LLC, MN) throughout the 55-d backgrounding period (Table 1).

418 At the end of backgrounding, all cattle were placed into a feedlot and delivered a similar finishing high energy ration until harvest. Between backgrounding to finishing, body weight

420 (BW) data were collected and average daily gain calculated. Calves were weighed using a hydraulic squeeze chute (Tru-Test XR 3000, Mineral Wells, TX) with load cells mounted under

422 the chute. Cattle had ad libitum access to water and free-choice minerals (Wind and Rain, Purina Animal Nutrition LLC, MN) throughout the 55-d backgrounding period and during finishing.

424 Average daily gain (ADG) was then calculated from periodic BW measurements during backgrounding and finishing phases.

\section{Rumen sample collection}

428 Samples were collected by using an esophageal tubing (Rumen-Mate ${ }^{\circledR}$, B \& B Manufacturing, Sumas, WA). During the entire study period, rumen ingesta samples were collected at weaning 430 (d 0; T1), early backgrounding (d 28; T2), late backgrounding (d 55; T3), early finishing (d 121; T4) and late finishing (d 185; T5). After collection, rumen samples were stored in carefully 432 labeled $50 \mathrm{~mL}$ plastic tubes and transported to the laboratory for storage in $-80{ }^{\circ} \mathrm{C}$ until DNA extraction. 
436 DNA was extracted by repeated bead-beating followed by precipitation, elution and purification using columns from the QIAamp ${ }^{\circledR}$ DNA PowerSoil Kit, (Germantown, MD). After DNA integrity

438 was measured, high quality DNA of 189 samples were used for rumen bacteria community profiling through $16 \mathrm{~S}$ rRNA amplicon sequencing, targeting the V4 hyper variable region (barcode

440 primer pair $515 \mathrm{f}$-GTGCCAGCMGCCGCGGTAA and 806rGGACTACHVGGGTWTCTAAT) on the Illumina MiSeq sequencing platform. Raw reads were trimmed to remove primers using

442 cutadapt, and filtered to remove low quality reads (less than $\mathrm{Q}=30$ ) using fastx_toolkit. High quality reads were considered for downstream analysis using the DADA2 plugin within

444 qiime2[49], which performs denoising, merging of paired-end reads and removal of chimeric sequences to produce unique amplicon sequence variants (ASVs). Taxonomic assignment of these

446 ASVs was carried out using the trained naïve Bayes classifier on reference sequences (clustered at 99\% sequence identity) from Greengenes 13_8 plugins within QIIME2.

\section{Statistical Analyses}

450 Average daily gain data was analyzed with the use of SAS 9.4 (SAS Inst., Inc., Cary, NC) as a completely randomized design with individual animals used as experimental units. Data were

452 checked for normality using PROC UNIVARIATE procedure of SAS (SAS Inst., Inc., Cary, $\mathrm{NC}$ ). Average daily gain data calculated from body weight measurements collected repeatedly

454 throughout the study and were analyzed as repeated measures using the PROC MIXED procedure of SAS (SAS Inst., Inc., Cary, NC). The model included the fixed effects of 456 backgrounding treatment, sampling $d$ and treatment $\mathrm{x}$ sampling $\mathrm{d}$ interaction while individual animals were considered a random effect. The $P$ values were adjusted for multiplicity based on 458 the Tukey-Kramer method. All microbial community ecology analyses were performed within 
the R statistical interface[50]. Alpha diversity (Shannon, Observed and Simpson) and beta

460 diversity (Bray Curtis distances) were calculated using the $\mathrm{R}$ vegan package[51]. Weighted and unweighted UniFRac distances were calculated using the phyloseq package[52]. Permutational

462 multivariate analyses of variance (PERMANOVA) test was used to check the significant differences. The false discovery rate (FDR)-adjusted Kruskal-Wallis multiple comparisons

$464(\mathrm{q}<0.05)$ and species indicator analysis (indicator values, $>0.5 ; P<0.05)$ as implemented in the labdsv R package ([53], were used to detect taxa differentially abundant at weaning (T1), 466 backgrounding (T2\&T3) and finishing (T4\&T5). All graphs were made using the Vegan and ggplots R package[54]. Association between average daily and representative genus was 468 measured using spearman correlation coefficients within the R psych package[55]. Network visualization and calculation of network attributes were carried out using Cytoscape.

\section{Abbreviations}

472 16S rRNA: 16 Svedberg ribosomal ribonucleic acid; ADG: Average daily gain; ANOVA: Analysis of variance; BLAST: Basic local alignment search tool; bp: Base pair; CC:

474 Cover crop; DMI: Dry matter intake; DL: Drylot; DNA: Deoxyribonucleic acid; ASV: Amplicon Sequence Variant; PP: Perennial pasture; T1: Weaning: T2: Early backgrounding; T3: Late 476 backgrounding: T4: Early finishing: T5: Late finishing

\section{Acknowledgements}

Sampling of ruminal samples was carried out at the Beef Cattle Research Farm, North Central 480 Research and Outreach Center (NCROC), University of Minnesota, Grand Rapids, MN from 2018 to 2019 . We thank the staff of NCROC for assisting with animal care and sampling. We

482 also thank personnel at the University of Minnesota Genomics Center (UMGC) for assisting in 
all sequencing procedures. The Data was processed using resources from the High-Performance

484 Computing Cluster of the University of Minnesota computing Institute (MSI).

(https://www.msi.umn.edu/).

486

Funding

488 Funding for this study was provided to AG and MW by the University of Minnesota's

Agricultural Research, Education, Extension and Technology Transfer Program (AGREETT)

490 and USDA experimental station, project number MN-16-122.

\section{Authors' contributions}

Experimental design by AG and MJW. Experimental work by BOO, SD, EJ, AG and MJW. Data

494 analysis was performed by BOO, AKS and AG, and the manuscript was written by BOO and AG. All authors read and approved the final manuscript.

496

Availability of data and materials

498 Raw 16S rRNA sequence data are available from the Nucleotide Archive under study accession number PRJNA763290.

500

\section{Ethics approval and consent to participate}

502 Rumen sampling was conducted at the Beef Cattle Research Farm, North Central Research and Outreach Center, University of Minnesota, Grand Rapids, MN from 2018 to 2019. The

504 experimental protocol was approved by the University of Minnesota Animal Care and Use Committee (approval number 1807-36177A). 


\section{Consent for publication}

508 Not applicable.

\section{Competing interests}

The authors declare that they have no competing interests.

512

\section{Author details}

514 +Department of Food and Animal Sciences, Alabama A\&M University, Normal, AL 35762, USA

$516++$ Current affiliation: Community Engagement \& Partnerships, Eastern West Virginia Community and Technical College, Moorefield, WV 26836, USA

$518{ }^{1}$ Department of Animal Science, University of Minnesota, Saint Paul, MN 5108, USA 


\section{References}

1. Godfray HCJ, Beddington JR, Crute IR, Haddad L, Lawrence D, Muir JF, et al. Food security: the challenge of feeding 9 billion people. Science. 2010;327:812-8.

2. Henchion M, Hayes M, Mullen A, Fenelon M, Tiwari B. Future Protein Supply and Demand: Strategies and Factors Influencing a Sustainable Equilibrium [Internet]. Foods. 2017. p. 53. Available from: http://dx.doi.org/10.3390/foods6070053

3. Desa U. United nations department of economic and social affairs, population division. world population prospects: The 2015 revision, key findings and advance tables. Technical Report. Working Paper No. ESA/P/WP. 241; 2015.

4. Pasiakos SM, Agarwal S, Lieberman HR, Fulgoni VL. Sources and Amounts of Animal, Dairy, and Plant Protein Intake of US Adults in 2007-2010. Nutrients. Multidisciplinary Digital Publishing Institute; 2015;7:7058-69.

5. Nkrumah JD, Okine EK, Mathison GW, Schmid K, Li C, Basarab JA, et al. Relationships of feedlot feed efficiency, performance, and feeding behavior with metabolic rate, methane production, and energy partitioning in beef cattle1 [Internet]. Journal of Animal Science. 2006. p. 145-53. Available from: http://dx.doi.org/10.2527/2006.841145x

6. Bergman EN. Energy contributions of volatile fatty acids from the gastrointestinal tract in various species. Physiol Rev. 1990;70:567-90.

7. Paz HA, Anderson CL, Muller MJ, Kononoff PJ, Fernando SC. Rumen Bacterial Community Composition in Holstein and Jersey Cows Is Different under Same Dietary Condition and Is Not Affected by Sampling Method. Front Microbiol. 2016;7:1206.

8. Li F, Zhou M, Ominski K, Guan LL. Does the rumen microbiome play a role in feed efficiency of beef cattle? J Anim Sci. Narnia; 2016;94:44-8.

9. Thomson DU, White BJ. Backgrounding Beef Cattle [Internet]. Veterinary Clinics of North America: Food Animal Practice. 2006. p. 373-98. Available from:

http://dx.doi.org/10.1016/j.cvfa.2006.03.010

10. Peel DS. Beef cattle growing and backgrounding programs. Vet Clin North Am Food Anim Pract. 2003;19:365-85, vi.

11. Perillat BJ, Brown WJ, Cohen RDH. A risk efficiency analysis of backgrounding and finishing steers on pasture in Saskatchewan, Canada. Agric Syst. 2004;80:213-33.

12. Kumar R, Lardner HA, McKinnon JJ, Christensen DA, Damiran D, Larson K. Comparison of alternative backgrounding systems on beef calf performance, feedlot finishing performance, carcass traits, and system cost of gain. The Professional Animal Scientist. Elsevier; 
2012;28:541-51.

13. Cox-O'Neill JL, Hales KE, Ulmer KM, Rasby RJ, Parsons J, Shackelford SD, et al. The effects of backgrounding system on growing and finishing performance and carcass characteristics of beef steers1 [Internet]. Journal of Animal Science. 2017. p. 5309-19. Available from: http://dx.doi.org/10.2527/jas2017.1934

14. Chibisa GE, Beauchemin KA. Effects of feeding corn silage from short-season hybrids and extending the backgrounding period on production performance and carcass traits of beef cattle. J Anim Sci. 2018;96:2490-503.

15. Lazzarotto EFC de O, de Oliveira Lazzarotto EFC, de Menezes LFG, Paris W, Molinete ML, Schmitz GR, et al. Backgrounding steers on temperate grasses mixed with vetch and/or using energy supplementation [Internet]. Asian-Australasian Journal of Animal Sciences. 2019. p. 800-7. Available from: http://dx.doi.org/10.5713/ajas.18.0603

16. Mulliniks JT, Hawkins DE, Kane KK, Cox SH, Torell LA, Scholljegerdes EJ, et al. Metabolizable protein supply while grazing dormant winter forage during heifer development alters pregnancy and subsequent in-herd retention rate. J Anim Sci. 2013;91:1409-16.

17. Summers AF, Weber SP, Lardner HA, Funston RN. Effect of beef heifer development system on average daily gain, reproduction, and adaptation to corn residue during first pregnancy1 [Internet]. Journal of Animal Science. 2014. p. 2620-9. Available from: http://dx.doi.org/10.2527/jas.2013-7225

18. St. Louis DG, Engelken TJ, Little RD, Edwards NC. Case study: Systems to reduce the cost of preconditioning calves. Professional animal scientist [Internet]. 2003;19. Available from: http://agris.fao.org/agris-search/search.do?recordID=US201300951894

19. Franzluebbers AJ, Stuedemann JA. Soil physical responses to cattle grazing cover crops under conventional and no tillage in the Southern Piedmont USA. Soil Tillage Res. 2008; 100:141-53.

20. Allmaras RR, Schomberg HH, Douglas CL, Dao TH. Soil organic carbon sequestration potential of adopting conservation tillage in US croplands. J Soil Water Conserv. Soil and Water Conservation Society; 2000;55:365-73.

21. Forsberg CW, Cheng K-J, White BA. Polysaccharide Degradation in the Rumen and Large Intestine. In: Mackie RI, White BA, editors. Gastrointestinal Microbiology: Volume 1 Gastrointestinal Ecosystems and Fermentations. Boston, MA: Springer US; 1997. p. 319-79.

22. Foster JA, Krone SM, Forney LJ. Application of ecological network theory to the human microbiome. Interdiscip Perspect Infect Dis. 2008;2008:839501.

23. Naqvi A, Rangwala H, Keshavarzian A, Gillevet P. Network-Based Modeling of the Human Gut Microbiome [Internet]. Chemistry \& Biodiversity. 2010. p. 1040-50. Available from: http://dx.doi.org/10.1002/cbdv.200900324 
24. Layeghifard M, Hwang DM, Guttman DS. Disentangling Interactions in the Microbiome: A Network Perspective. Trends Microbiol. 2017;25:217-28.

25. Golbeck J. Chapter 3 - Network Structure and Measures. In: Golbeck J, editor. Analyzing the Social Web. Boston: Morgan Kaufmann; 2013. p. 25-44.

26. Ren Y, Ay A, Kahveci T. Shortest path counting in probabilistic biological networks. BMC Bioinformatics. 2018;19:465.

27. Flint HJ, Bayer EA, Rincon MT, Lamed R, White BA. Polysaccharide utilization by gut bacteria: potential for new insights from genomic analysis. Nat Rev Microbiol. Nature Publishing Group; 2008;6:121.

28. Drouillard JS, Kuhl GL. Effects of previous grazing nutrition and management on feedlot performance of cattle. J Anim Sci. 1999;77 Suppl 2:136-46.

29. Bevans DW, Beauchemin KA, Schwartzkopf-Genswein KS, McKinnon JJ, McAllister TA. Effect of rapid or gradual grain adaptation on subacute acidosis and feed intake by feedlot cattle. J Anim Sci. 2005;83:1116-32.

30. Durunna ON, Mujibi FDN, Goonewardene L, Okine EK, Basarab JA, Wang Z, et al. Feed efficiency differences and reranking in beef steers fed grower and finisher diets. J Anim Sci. 2011;89:158-67.

31. Petri RM, Schwaiger T, Penner GB, Beauchemin KA, Forster RJ, McKinnon JJ, et al. Changes in the rumen epimural bacterial diversity of beef cattle as affected by diet and induced ruminal acidosis. Appl Environ Microbiol. 2013;79:3744-55.

32. Malmuthuge N, Li M, Chen Y, Fries P, Griebel PJ, Baurhoo B, et al. Distinct commensal bacteria associated with ingesta and mucosal epithelium in the gastrointestinal tracts of calves and chickens. FEMS Microbiol Ecol. 2012;79:337-47.

33. Petri RM, Schwaiger T, Penner GB, Beauchemin KA, Forster RJ, McKinnon JJ, et al. Characterization of the core rumen microbiome in cattle during transition from forage to concentrate as well as during and after an acidotic challenge. PLoS One. 2013;8:e83424.

34. Hernandez-Sanabria E, Goonewardene LA, Wang Z, Zhou M, Moore SS, Guan LL. Influence of sire breed on the interplay among rumen microbial populations inhabiting the rumen liquid of the progeny in beef cattle. PLoS One. 2013;8:e58461.

35. Granja-Salcedo YT, Fernandes RM, de Araujo RC, Kishi LT, Berchielli TT, de Resende FD, et al. Long-Term Encapsulated Nitrate Supplementation Modulates Rumen Microbial Diversity and Rumen Fermentation to Reduce Methane Emission in Grazing Steers. Front Microbiol. 2019;10:614.

36. Myer PR, Freetly HC, Wells JE, Smith TPL, Kuehn LA. Analysis of the gut bacterial communities in beef cattle and their association with feed intake, growth, and efficiency. J Anim Sci. 2017;95:3215-24. 
37. Ren H, Su X, Bai H, Yang Y, Wang H, Dan Z, et al. Specific enrichment of microbes and increased ruminal propionate production: the potential mechanism underlying the high energy efficiency of Holstein heifers fed steam-flaked corn. AMB Express. 2019;9:209.

38. Bryant MP, Small N. Characteristics of two new genera of anaerobic curved rods isolated from the rumen of cattle. J Bacteriol. 1956;72:22-6.

39. O'Herrin SM. Characteristics of Substrate Utilization and Metabolism by the Ruminal Anaerobes Succinivibrio Dextrinosolvens and Succinimonas Amylolytica. University of Wisconsin--Madison; 1993.

40. O’Herrin SM, Kenealy WR. Glucose and carbon dioxide metabolism by Succinivibrio dextrinosolvens. Appl Environ Microbiol. 1993;59:748-55.

41. Hornick JL, Van Eenaeme C, Gérard O, Dufrasne I, Istasse L. Mechanisms of reduced and compensatory growth. Domest Anim Endocrinol. 2000;19:121-32.

42. Stevenson DM, Weimer PJ. Dominance of Prevotella and low abundance of classical ruminal bacterial species in the bovine rumen revealed by relative quantification real-time PCR [Internet]. Applied Microbiology and Biotechnology. 2007. p. 165-74. Available from: http://dx.doi.org/10.1007/s00253-006-0802-y

43. Shabat SKB, Sasson G, Doron-Faigenboim A, Durman T, Yaacoby S, Berg Miller ME, et al. Specific microbiome-dependent mechanisms underlie the energy harvest efficiency of ruminants [Internet]. The ISME Journal. 2016. p. 2958-72. Available from:

http://dx.doi.org/10.1038/ismej.2016.62

44. Carrillo JA, He Y, Li Y, Liu J, Erdman RA, Sonstegard TS, et al. Integrated metabolomic and transcriptome analyses reveal finishing forage affects metabolic pathways related to beef quality and animal welfare. Sci Rep. 2016;6:25948.

45. Chiquette J, Allison MJ, Rasmussen MA. Use of Prevotella bryantii 25A as a Probiotic to Reduce the Risk of Ruminal Acidosis in Dairy Cows. Animal Industry Report. 2008;654:50.

46. Li F, Hitch TCA, Chen Y, Creevey CJ, Guan LL. Comparative metagenomic and metatranscriptomic analyses reveal the breed effect on the rumen microbiome and its associations with feed efficiency in beef cattle. Microbiome. 2019;7:6.

47. Li F, Guan LL. Metatranscriptomic Profiling Reveals Linkages between the Active Rumen Microbiome and Feed Efficiency in Beef Cattle. Appl Environ Microbiol [Internet]. 2017;83. Available from: http://dx.doi.org/10.1128/AEM.00061-17

48. Paz HA, Hales KE, Wells JE, Kuehn LA, Freetly HC, Berry ED, et al. Rumen bacterial community structure impacts feed efficiency in beef cattle. J Anim Sci. 2018;96:1045-58.

49. Bolyen E, Rideout JR, Dillon MR, Bokulich NA, Abnet CC, Al-Ghalith GA, et al. Author Correction: Reproducible, interactive, scalable and extensible microbiome data science using QIIME 2. Nat Biotechnol. 2019;37:1091. 
50. Pinheiro J, Bates D, DebRoy S, Sarkar D. R Core Team (2017) nlme: linear and nonlinear mixed effects models. R package version 3.1-131. Computer software] Retrieved from https://CRAN R-project org/package= nlme. 2017;

51. Okasanen J, Blanchet FG, Kindet R, Legendre P, Minchin PR, O'Hara RB, et al. vegan: Community Ecology Package, R package version 2.0-4. 2012.

52. $\mathrm{Pj} \mathrm{M}$. Holmes S. phyloseq: an R package for reproducible interactive analysis and graphics of microbiome census data. PLoS One. 2013;8:e61217.

53. Roberts DW. labdsv: ordination and multivariate analysis for ecology. 2016. R package version. 2016;1-4.

54. Wickham H, Chang W, Others. ggplot2: An implementation of the Grammar of Graphics. R package version 0 7, URL: http://CRAN R-project org/package= ggplot2. 2008;3.

55. Revelle W. An overview of the psych package. Department of Psychology Northwestern University Accessed on March. Citeseer; 2011;3:1-25. 


\section{Supplementary Files}

This is a list of supplementary files associated with this preprint. Click to download.

- BKGSMtextandFigures.pdf

- BKGTableS1.xIsx 